\title{
Acknowledgement to Reviewers of Diagnostics in 2018
}

\author{
Diagnostics Editorial Office
}

MDPI, St. Alban-Anlage 66, 4052 Basel, Switzerland

Published: 9 January 2019

Rigorous peer-review is the corner-stone of high-quality academic publishing. The editorial team greatly appreciates the reviewers who contributed their knowledge and expertise to the journal's editorial process over the past 12 months. In 2018, a total of 85 papers were published in the journal, with a median time to first decision of 17 days and a median time to publication of 36 days. The editors would like to express their sincere gratitude to the following reviewers for their cooperation and dedication in 2018:

\author{
Abuzaid, Ahmed \\ Adhikari, Prakash \\ Ahmed, Aamir \\ Alian, Aymen \\ Armstrong, Glen \\ Ashish, Pathak \\ Banitsas, Konstantinos A. \\ Bauckneht, Matteo \\ Bechsgaard, Rie Eriksen \\ Bellani, Marcella \\ Bender, Hans-Ulrich \\ Bernardello, Fabio \\ Bernassau, Anne \\ Bernemann, Christof \\ Bharaj, Preeti \\ Blyth, Benjamin J. \\ Board, Mary \\ Bordonaro, Michael \\ Brewer Gutierrez, Olaya \\ Cai, Lisheng \\ Carding, Simon \\ Carpentieri, David \\ Casals Terre, Jasmina \\ Cascini, Giuseppe Lucio \\ Catry, Boudewijn \\ Cavagnaro, Marta \\ Chalah, Moussa \\ Chatterjee, Jhinuk \\ Chen, Wenchun \\ Choi, Yun-Jung \\ Clancy, Eoin \\ Clauser, Paola
}

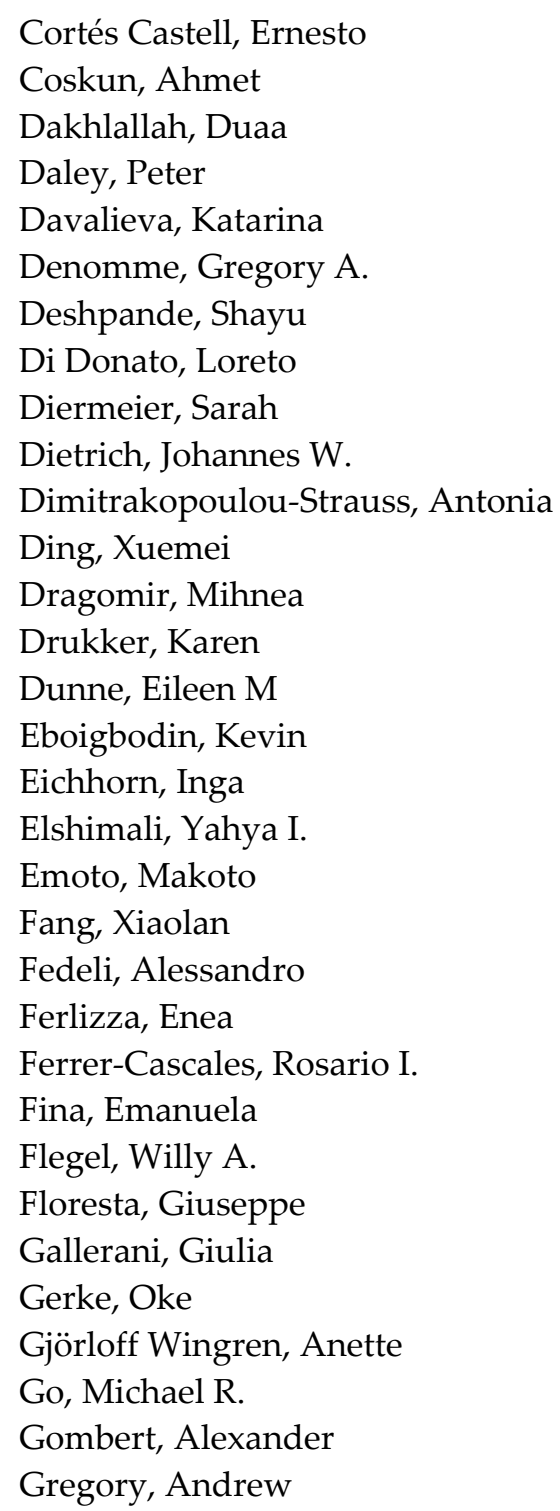


Guan, Weihua

Gupta, Rishein

Gupta, Vijayalaxmi

Hamad-Schifferli, Kimberli

Han, Seung Hee

Helbig, Marko

Hendrikse, Harry

Hingorani, Dina

Hossain, Md. Delwar

Hsieh, Kuangwen

Hsu, Sung-Po

$\mathrm{Hu}$, Xiaosu

Huffman, Mark

Ierardi, Enzo

Iinuma, Hisae

Islam, Md Asiful

Isola, Gaetano

Jaiswal, Ashvin R.

Jason, Fiering

Jeong, Keun-Yeong

Jeschke, Udo

Jiménez-Bonilla, Julio Francisco

Joshi, Bharat

Kairemo, Kalevi

Kaushik, Ajeet

Kerr, Jonathan

Khoo, Bee Luan

Kim, Nam-Young

Klein, Katherine A.

Knippa, Emily

Kotsiantis, Sotiris

Kottner, Jan

Koutsoupidou, Maria

Krishna, Somashekar G.

Kumar, Suneel

Kung, Yu-Chun

Landsheer, Hans

Le Pichon, Jean-Baptiste

Lehmann-Chehe, Jacqueline

Lenzo, Nat

Leon Mateos, Luis

Lewis, Mark

$\mathrm{Li}$, Lin

Li, Xiang-Guo

Lidbury, Brett

Lima, Ivan

Liu, Wei-Min

Liu, Yu

Loades, Maria

Løbner-Olesen, Anders

Lodi, Matteo Bruno

Losurdo, Giuseppe
Lum, Ying Wei

Lyon, Gholson

Ma, Michelle

Mainprize, James

Margolies, Laurie

Marín, Mercedes

Martins, Ian James

Masarone, Daniele

Mavridis, Konstantinos

Mayo, Ray

Mcmorrow, Tara

Mejzlik, Jan

Mercado-Shekhar, Karla P.

Minaga, Kosuke

Mirza, Aleem KH

Mohd Shah, Syaiful Redzwan

Möhlendick, Birte

Moncayo, Roy

Morton, Ben

Moseley, Tanya

Moxon, Joseph

Nacul, Luis C

Nery, Fabio

Nima, Zeid A.

Norcic, Gregor

Nyström, Pär

Olivito, Giusy

Omura, Yasuhisa

Osaki, Takako

Ozen, Mehmet Ozgun

Page, Anne-Laure

Papacleovoulou, Georgia

Parsons, Lauren N.

Pasian, Marco

Pastey, Manoj

Perez, Mauricio

Petersen, Lars

Popovic, Milica

Porter, Emily

Potus, François

Povlsen, Sebastian

Qasaimeh, Mohammad A.

Qiu, Hao

Qiu, Zhen

Rahib, Lola

Rahimi, Farid

Rainger, George Edward

Ramalingam, Naveen

Ramchandani, Divya

Reif, Kathryn E.

Ritter, Kerstin

Rojo-Álvarez, José Luis 
Rosenzweig, Allison

Rydosz, Artur

San Miguel, Adriana

Sankarasubramanian, Vishwanath

Saponara, Sergio

Saraste, Antti

Sathe, Gajanan Jalindarnath

Sathekge, Mike

Sato-Bigbee, Carmen

Sciacchitano, Salvatore

Serafini, Gianluca

Serefko, Anna

Shah, Jyotsna

Shao, Dangdang

Shi, Jiahai

Shiao, Young-Ji

Shim, Hyo Sup

Singh, Virtaj

Smith, Heather F.

Soares, Paula

Song, Yong-Ak (Rafael)

Sorensen, Karina Dalsgaard

Speight, Nigel

Sperber, Geoffrey H.

Spizzo, Gilbert

Sreerama, Subramanya

Staines, Donald

Strand, Sven-Erik

Taguchi, Y-h.
Takayama, Kenichi

Tan, Isabella

Tcheslavski, Gleb V.

Teo, Soo Kng

Terracciano, Daniela

Theodorsson, Elvar

Tsukamoto, Tetsuya

Twisk, Frank

Upadhyay, Abhinav

Uwiera, Trina

VanderWeele, David

Vashist, Arti

Vasilevsky, Nicole

Vaughan, Christopher L.

Venkataraman, Rajesh

Vijayaraghavan, Gopal R.

Vitorino, Rui

Wei, Changyong

Wilczek, Brigitte

Xia, Hui

$\mathrm{Xu}$, Zhihuo

Ye, Yanqi

Youssofzadeh, Vahab

Zacho, Helle D

Zagidullin, Naufal

Zakaria, Amer

Zun, Zungho

(C) 2019 by the authors. Submitted for possible open access publication under the

terms and conditions of the Creative Commons Attribution (CC-BY) license (http://creativecommons.org/licenses/by/4.0/). 